\title{
Mejora de la calidad de servicio ofrecido en las mypes de hospedaje ubicadas en destinos emergentes del Perú utilizando el Sistema de Aplicación de Buenas Prácticas
}

SusAn Herrera Marín ${ }^{1}$

RECIBIDO: 08/11/2018 AcEPTADO: 07/01/2019

\begin{abstract}
RESUMEN
El presente artículo es el resultado de la necesidad de lograr que las mypes en el rubro de alojamiento mejoren la calidad de sus servicios a un coste adecuado. El Sistema de Aplicación de Buenas Prácticas (SABP) es una herramienta para las mypes, ya que favorece a la mejora de la calidad en los servicios ofrecidos, además los ayuda a posicionarse, aprovechando su zona y el destino de ubicación, para optimizar las condiciones de vida de su comunidad. De esta forma, en esta investigación se propone una metodología de aplicación que se ajusta al progreso de dichos negocios, para los cuales se busca el equilibrio entre la adecuada gestión del proceso administrativo, del servicio, de la infraestructura y equipamiento, del desempeño de colaboradores y del apoyo de áreas de soporte.
\end{abstract}

Palabras-claves: Buenas prácticas; calidad de servicio; mype; alojamiento; destino.

\section{INTRODUCCIÓN}

Una de las fuentes más importantes para el desarrollo de las ciudades a nivel mundial es el turismo. En ese sentido, el Perú se ha convertido en un destino relevante para esta actividad, ya que cuenta con aquellos recursos que pueden ser explotados de manera racional y sostenible. Además, el turismo puede brindar satisfacciones tanto a los propietarios de pequeños negocios como a todos aquellos actores que de manera directa o indirecta se relacionan con la ejecución de esta actividad económica. Así, sobre este rubro, Cabeza (2001) señala que "los expertos coinciden en que el turismo, conjuntamente con las comunicaciones y la informática, serán los tres sectores que regirán la economía mundial del tercer milenio, caracterizado por una penetración de la tecnología en todos los aspectos de la vida" (p. 257). Además, la Organización Mundial del Turismo (OMT) en su informe Turismo: Panorama 2020 (2002) afirma que se prevé que los ingresos provenientes del turismo internacional (excluido el transporte) alcancen los 2 billones de dólares en 2020 y que se gastará más de 5000 millones de dólares cada día, mientras que el número de llegadas de turistas internacionales en todo el mundo aumentará hasta casi 1600 millones en 2020. Asimismo, Francisco (2014) menciona que "las principales zonas de crecimiento, en lo que a inversiones extranjeras se refiere, se están trasladando a China, países latinoamericanos seguros y emergentes $y$, por último, a países africanos con las mismas condiciones" (p. 30).

La presente investigación se centrará en uno de los principales problemas que el turismo conlleva, el cual surge con la aparición de prestadores de servicios turísticos en los destinos emergentes del Perú, pues no siempre operan un producto o servicio de calidad. Por ese motivo, requieren especial atención y necesitan hacer llegar estos sistemas adaptados a sus micro y pequeñas empresas en este tipo de destinos, haciendo de su conocimiento, las directrices sobre la calidad en el sector.

Se propone el uso del Sistema de Aplicación de Buenas Prácticas (SABP) para las mypes en el sector hospedaje preexistente y en el que se hace uso de un instrumento de medición, el mismo

\footnotetext{
Licenciada en Administración de Turismo, con maestría en Gestión de Operaciones y Servicios Logísticos, por la Universidad Nacional Mayor de San Marcos. Posee conocimiento en calidad de servicios turísticos y hoteleros, tiene experiencia en docencia y actualmente es asistente de la bolsa de trabajo de Turismo y Hotelería de la Universidad de San Martín de Porres. Lima, Perú. E-mail: susan.herrera@unmsm.edu.pe
} 
que se ha replanteado a fin de convertirlo en una herramienta ágil en su aplicación y más acorde a la realidad de los destinos que aún no cuentan con desarrollo o cuentan con una incipiente actividad turística. Se tiene como base el Plan Estratégico Nacional de Turismo del Perú (PENTUR), el cual declara, sobre el turismo peruano a futuro, que se encuentra "elaborado con metas hacia el 2025, que cuenta con un modelo de desarrollo turístico, siendo uno de sus 4 pilares la diversificación y consolidación de la oferta" (Ministerio de Comercio Exterior y Turismo, 2016, párr. 6).

A partir de lo antes expuesto, se puede indicar que la finalidad de esta propuesta es brindar a los empresarios, gobiernos locales y regionales una herramienta para el logro del mejoramiento de la calidad de servicio en establecimientos de hospedaje. Ello sucede con el fin de poder tomar en cuenta el antes y durante la planificación y la operación de un establecimiento de este tipo, además de poder asegurar la ejecución de servicios de calidad generando un mayor flujo de visitantes tanto internos como receptivos y, a su vez, para que ello impacte positivamente en el entorno de la ubicación de estos negocios.

\section{Antecedentes}

La calidad ha sido un elemento predominante en la vida del hombre en todo su proceso evolutivo, siempre buscando el mayor beneficio con un menor esfuerzo. Hacia 1920, la calidad inició con cambios más sustanciales, los mismos que se resumen a continuación en la Tabla 1:
"Un avance significativo, dentro del concepto de calidad, es el hecho de incorporar al cliente como un actor fundamental [...]. La calidad no es un resultado final sino [...] un camino sin final, un sendero que siempre es necesario recorrer" (López et al., 2018, p. 444). De esa forma, la gestión de esta asume la calidad como un medio para alcanzar propósitos o como un horizonte organizacional que orienta la acción, reconociendo tanto aspectos predecibles como elementos aleatorios, impredecibles e inesperados (Sanabria, Romero y Flórez, 2014). La calidad es una realidad inacabable, ya que se trata de un camino por donde siempre debemos andar para lograr los objetivos trazados en torno al progreso de las mypes, al cliente y su plenitud.

De esta manera, la Organización de las Naciones Unidas para la Alimentación y la Agricultura (2015) señala que "una buena práctica no es tan solo una práctica que se define buena en sí misma, sino que se ha demostrado y validado que funciona bien y produce buenos resultados y, por lo tanto, se recomienda como modelo" (p. 1). Por otro lado, la Organización de las Naciones Unidas para la Educación, la Ciencia y la Cultura (2017), en el marco de su programa Management of Social Transformations (MOST), define que buenas prácticas deben ser innovadoras, efectivas, sostenibles y replicables, puesto que son recursos para el accionar en el presente.

Asimismo, se pueden llamar "sistemas a todas aquellas referencias, como códigos, normas o modelos, que proporcionan directrices, orientaciones o

Tabla 1. Evolución del concepto de calidad.

\begin{tabular}{|c|c|c|c|}
\hline Etapas & Concepto & Finalidad & Contexto histórico \\
\hline $\begin{array}{c}\text { Control de calidad } \\
\text { por inspección }\end{array}$ & $\begin{array}{c}\text { Uso de técnicas de control de } \\
\text { calidad por inspección y métodos } \\
\text { estadísticos que permiten identificar } \\
\text { los productos defectuosos. }\end{array}$ & $\begin{array}{c}\text { Satisfacer estándares y condiciones } \\
\text { técnicas de un producto. }\end{array}$ & $\begin{array}{c}\text { Finales de la Primera } \\
\text { Guerra Mundial }\end{array}$ \\
\hline $\begin{array}{c}\text { Aseguramiento de } \\
\text { calidad }\end{array}$ & $\begin{array}{c}\text { Se asegura la calidad sin escati- } \\
\text { mar en costos, pero garantizando } \\
\text { mayor volumen de producción en } \\
\text { menor tiempo. }\end{array}$ & $\begin{array}{c}\text { Garantizar un producto eficaz en canti- } \\
\text { dades y tiempos solicitados. }\end{array}$ & $\begin{array}{c}\text { Segunda Guerra Mun- } \\
\text { dial }\end{array}$ \\
\hline $\begin{array}{c}\text { Calidad total } \\
\text { para evitar productos defectuosos } \\
\text { en todas las áreas funcionales de } \\
\text { la organización. }\end{array}$ & $\begin{array}{c}\text { Satisfacer al cliente evitando errores. } \\
\text { Generar competitividad con el menor } \\
\text { costo y la mayor participación de em- } \\
\text { pleados. }\end{array}$ & Década de los 70 y los \\
90
\end{tabular}

Fuente: Elaboración propia a partir del trabajo de Cubillos y Rozo (2009). 
requerimientos referentes al modo cómo ha de gestionarse una organización y/o prestarse un servicio" (Barbero, 2007, p. 5). Conexión ESAN (25 de mayo de 2016), en un artículo titulado "Marketing de servicios: significado y características", menciona que las principales características de los servicios son la intangibilidad, la heterogeneidad/variabilidad, el ser perecedero y la inseparabilidad/simultaneidad de producción y consumo.

\section{Sistema de Aplicación de Buenas Prácticas (SABP)}

El Ministerio de Comercio Exterior y Turismo del Perú (MINCETUR) (2017) indica que

El Plan Nacional de Calidad Turística del Perú (CALTUR) es un instrumento de gestión del Sector Turismo que establece las estrategias y líneas de acción en materia de calidad, para posicionar al Perú como un destino global de calidad, logrando que sus destinos y productos sean percibidos como espacios y experiencias de alto valor en todos sus componentes (párr. 1).

EI SABP es la herramienta del CALTUR que permite insertar a los prestadores de servicios turísticos en procesos de mejora continua, la cual es aplicada por el Centro de Formación en Turismo (CENFOTUR). Además, está dirigida principalmente a los rubros de hospedaje, restaurante y servicios afines, agencias de viajes y turismo.

De acuerdo con el Decreto Supremo N. ${ }^{\circ}$ 001-2015-MINCETUR del Reglamento de establecimientos de hospedaje (9 de junio de 2015), estos se clasifican como: hotel, 1 a 5 estrellas; apart-hotel, 3 a 5 estrellas; hostal, 1 a 3 estrellas y albergue, sin categoría (art. 3). Además, es importante definir los cuatro aspectos a verificar que se desarrollan en el instrumento usado para medir la calidad de los establecimientos de hospedaje según el SABP y que es razón de la presente investigación, el cuestionario para establecimientos de hospedaje (fase de evaluación del SABP):

Proceso estratégico. Establecido por áreas de estrategia, gestión y de back office. Se halla dividida en administración, marketing y ventas, y logística.

Proceso principal. Constituido por cuatro pilares de la operación: recepción y reservas (infraestructura y equipamiento), recepción y reservas (gestión del servicio), housekeeping (infraestructura y equipamiento) y housekeeping (gestión del servicio).
Proceso de soporte. Conformado por alimentos y bebidas, mantenimiento y seguridad.

Competencias de los colaboradores. Compuesto por uno de los aspectos más importantes de la organización.

\section{METODOLOGÍA}

La muestra está determinada por trece establecimientos de hospedaje de la región Ica. El procedimiento usado para la selección de la muestra fue el método probabilístico y el muestreo es al azar simple. Además, las técnicas de recolección de datos usadas fueron:

Entrevistas y encuestas elaboradas a los microempresarios del rubro hotelero en la localidad de Ica (cuestionario SABP y cuestionario propuesto).

Investigación de gabinete, revisión de los diversos datos históricos de la empresa relacionados con las variables de investigación.

Análisis de contenido cuantitativo realizada mediante el uso del programa computarizado de análisis estadístico Minitab 17.

\section{RESULTADOS}

\section{Reformulación y creación del nuevo cuestionario propuesto}

Para la elaboración del nuevo cuestionario, se trabajó con base en la muestra del momento "antes", usando Minitab 17 para ir identificando los ítems menos relevantes y evaluar la calidad del establecimiento. De este modo, se logró ello a través de la comparación del resultado total o nivel de calidad alcanzado por la empresa versus cada resultado subtotal o puntaje alcanzado en cada una de las partes que contienen dicho cuestionario y estas a su vez con sus subpartes. A continuación, el número de ítems que contiene el cuestionario inicial hasta el cuestionario propuesto se muestra en la Tabla 2.

Como se muestra en la tabla anterior, el cuestionario inicial o Asys contaba con 180 ítems, mientras que el cuestionario To be contaba con 131 puntos. Luego de aplicar Minitab 17 a los porcentajes de los puntajes de las muestras tomadas y descartar dichos ítems basándose en el conocimiento previo del autor. Asimismo, en la versión To be mejorado, se han incluido o modificado 7 nuevos aspectos a evaluar, resultando el nuevo cuestionario propuesto con un total de 138 ítems. 


\section{Resultados de la aplicación de cuestionarios}

En seguida, se muestran los resultados de la aplicación de los dos cuestionarios, en el momento "2016 (antes)", donde se hizo uso del cuestionario Asys, y en el momento "2017 (después)", en el que se hizo uso del cuestionario propuesto. Los datos se muestran en la Tabla 3:

De esta forma, se observa que las empresas han tenido una mejora en su puntuación entre las dos intervenciones, lo que demuestra que el nuevo cues- tionario implementado obtuvo buenos resultados y alcanzó el objetivo planificado en la investigación.

\section{Prueba de hipótesis}

\subsection{Hipótesis general}

Inicialmente se realizó el análisis de normalidad a través de la prueba de Anderson-Darling, la cual es la más efectiva en la detección de no normalidad en las colas de la distribución, pues estas últimas son las más críticas dentro de la distribución. Asimismo,

Tabla 2. Criterios de calidad en cuestionarios.

\begin{tabular}{|c|c|c|c|c|}
\hline \multirow{2}{*}{ Dimensiones } & \multirow{2}{*}{ Subdimensiones } & \multicolumn{3}{|c|}{ Criterios } \\
\hline & & Asys & To be & To be mejorado \\
\hline \multirow[t]{3}{*}{ La gestión del proceso estratégico } & Administración & 12 & 8 & 0 \\
\hline & Marketing y ventas & 6 & 6 & 0 \\
\hline & Logística & 7 & 5 & 0 \\
\hline \multirow[t]{4}{*}{ La gestión del proceso principal } & $\begin{array}{l}\text { Recepción y reservas (infraestructura y equipa- } \\
\text { miento) }\end{array}$ & 18 & 15 & 2 \\
\hline & Recepción y reservas (gestión del servicio) & 28 & 26 & 1 \\
\hline & Housekeeping (infraestructura y equipamiento) & 63 & 46 & 0 \\
\hline & Housekeeping (gestión del servicio) & 20 & 17 & 0 \\
\hline \multirow[t]{3}{*}{ La gestión del proceso de soporte } & Alimentos y bebidas & 9 & 1 & 2 \\
\hline & Mantenimiento & 2 & 0 & 1 \\
\hline & Seguridad & 8 & 1 & 1 \\
\hline $\begin{array}{l}\text { La gestión de las competencias de } \\
\text { los colaboradores }\end{array}$ & Colaboradores & 7 & 6 & 0 \\
\hline \multicolumn{2}{|l|}{ TOTAL } & 180 & 131 & 7 \\
\hline
\end{tabular}

Fuente: Elaboración propia.

Tabla 3. Resultados aplicación de cuestionarios.

\begin{tabular}{|c|l|c|c|c|c|}
\cline { 3 - 6 } \multicolumn{2}{c|}{} & \multicolumn{2}{c|}{2016 (antes) } & \multicolumn{2}{c|}{ 2017 (después) } \\
\hline $\mathbf{N} .^{\circ}$ & Establecimiento & Puntaje obtenido & Calificación & Puntaje obtenido & Calificación \\
\hline 1 & Hotel Suite Grau & $70,07 \%$ & En mejora & $76,69 \%$ & En mejora \\
\hline 2 & Hotel Duna Dorada & $81,91 \%$ & Aceptable & $89,19 \%$ & Aceptable \\
\hline 3 & Hostal Lindo Perú & $66,69 \%$ & En mejora & $74,25 \%$ & En mejora \\
\hline 4 & Hostal Antonio's & $66,03 \%$ & En mejora & $73,12 \%$ & En mejora \\
\hline 5 & Hotel Continental's & $60,29 \%$ & En mejora & $69,36 \%$ & En mejora \\
\hline 6 & Hostal Evelyn & $73,90 \%$ & En mejora & $76,03 \%$ & En mejora \\
\hline 7 & Hotel El Parral Inn & $78,31 \%$ & En mejora & $80,26 \%$ & Aceptable \\
\hline 8 & Hostal Oasis & $75,07 \%$ & En mejora & $79,89 \%$ & Aceptable \\
\hline 9 & Hostal Monarca & $77,35 \%$ & En mejora & $78,01 \%$ & En mejora \\
\hline 10 & Hostal Campo Alegre & $74,41 \%$ & En mejora & $77,16 \%$ & En mejora \\
\hline 11 & Hostal Vega's & $65,59 \%$ & En mejora & $78,29 \%$ & En mejora \\
\hline 12 & Hotel El Embrujo & $80,44 \%$ & Aceptable & $81,86 \%$ & Aceptable \\
\hline 13 & Hostal El Médano & $79,04 \%$ & En mejora & $79,61 \%$ & Aceptable \\
\hline & Promedios & $73,01 \%$ & & $77,98 \%$ & \\
\hline
\end{tabular}

Fuente: Elaboración propia. 
es importante mencionar que se está contando con una confiabilidad del $95 \%$ de concluir y los datos siguen una distribución normal. Las principales cifras obtenidas de esta prueba para cada momento (antes y después) se muestran en la Tabla 4:

Tabla 4. Resultados de análisis de normalidad.

\begin{tabular}{|l|c|c|}
\hline & Antes & Después \\
\hline A-Cuadrado & 0,35 & 0,19 \\
\hline P-Value & 0,425 & 0,885 \\
\hline Media & 0,73008 & 0,77975 \\
\hline
\end{tabular}

Fuente: Elaboración propia.

Se muestra que la distribución de los datos de la calidad en ambos momentos de aplicación tiene un comportamiento normal (P-Value $>0,05)$. De la misma forma, se determinó que la variable independiente "Sistema de Aplicación de Buenas Prácticas" es discreta y la variable dependiente "Mejora de la calidad de servicio de las mypes de hospedaje" es continua, por lo que se debe realizar el Two-Sample T-Test con Minitab 17. Luego se procedió con el análisis de Two-Sample T-Test, a partir del cual se concluyó que la implementación de la variable independiente afectó a la variable dependiente porque el $\mathrm{P}$-Value $<0,05$, debido a lo cual se demuestra la hipótesis alternativa.

\subsection{Hipótesis específica}

Se determinó que las dimensiones de la variable independiente (la gestión del proceso estratégico, la gestión del proceso principal, la gestión del proceso de soporte y la gestión de las competencias de los colaboradores) son continuas. De la misma forma, se encontró que la variable dependiente "Mejora de la calidad de servicio de las mypes de hospedaje" es continua, por lo cual se realizaron las pruebas de correlación y regresión con Minitab 17.

Análisis de correlación y regresión. Sirve para medir la fuerza y la dirección de la asociación entre dos variables continuas. En este caso, se usó el método de correlación de Pearson, mientras que el análisis de regresión se utilizó para estudiar la relación entre variables o factores cuantitativos referidos a un mismo grupo de unidades observadas.
Los principales datos de esta prueba aplicada para cada una de las hipótesis específicas se muestran en la Tabla 5.

A partir de la Tabla 5 se concluye que la gestión del proceso estratégico es significativa y afecta en un $53,78 \%$ a la calidad de servicio "antes", asimismo existe una relación fuerte entre ellos; la gestión del proceso principal es significativa y afecta en un $97,89 \%$ a la calidad de servicio "antes", asimismo existe una relación fuerte entre ellos; la gestión del proceso de soporte no es significativa y no afecta a la calidad de servicio "antes", asimismo no existe una relación entre ellos; y la gestión de la competencia de los colaboradores es significativa y afecta en un $43,78 \%$ a la calidad de servicio "antes", asimismo existe una relación media alta entre ellos.

\section{Informe de resumen de calidad en el momento "antes"}

Análisis de correlación y regresión. Se realizó el análisis de correlación de las cuatro dimensiones con respecto a la calidad del servicio "antes", en el cual se observa que la dimensión gestión del proceso de soporte tiene un coeficiente de Pearson cercano a 0 y el $\mathrm{P}$-Value $>0,05$ (igual a 0,999), lo que significa que esta dimensión no tiene relación con el problema.

Además, luego del análisis de regresión de las tres dimensiones restantes (gestión del proceso estratégico, gestión del proceso principal y gestión de las competencias de los colaboradores) versus la calidad del servicio "antes", se concluye que solamente esas tres dimensiones son significativas y afectan en conjunto en un $99,53 \%$ a la calidad del servicio, porque el $\mathrm{P}$-Value $<0,05$ y el $\mathrm{R}^{2}$ es $99,53 \%$. Este es el motivo por el que se descarta la dimensión gestión del proceso de soporte.

Por lo tanto, la ecuación que representa la mejora de la calidad de servicio de las mypes de hospedaje, en función a estas tres dimensiones, es la siguiente:

CALIDAD = 0,0302 + 0,1860 I. - Proceso estratégico + 0,6333 II. Proceso principal + 0,1222 IV. - Competencias de los colaboradores

Tabla 5. Resultados de análisis de correlación y regresión.

\begin{tabular}{|c|c|c|c|c|c|}
\hline & & Hipótesis 1 & Hipótesis 2 & Hipótesis 3 & Hipótesis 4 \\
\hline \multirow{2}{*}{ Correlación } & Coeficiente de Pearson & 0,733 & 0,989 & 0 & 0,662 \\
\cline { 2 - 6 } & P-Value & 0,004 & 0,000 & 0,999 & 0,014 \\
\hline \multirow{2}{*}{ Regresión } & R-Cuadrado & $53,78 \%$ & $97,89 \%$ & $0 \%$ & $43,78 \%$ \\
\hline
\end{tabular}

Fuente: Elaboración propia. 


\section{IMPACTOS}

\section{Propuesta para la solución del problema}

La solución que se plantea es la aplicación del sistema usando el nuevo cuestionario propuesto, el cual permitirá la mejora de la calidad del servicio de las mypes de hospedaje ofrecido en los destinos emergentes en el Perú, haciendo uso de menores recursos de tiempo y dinero, lo cual optimizará la aplicación del sistema. También, la implementación de este sistema puede ser financiado por los gobiernos regionales o locales, como las más de 15 regiones principales que reciben transferencias por canon minero. En un artículo de Gestión (1 de agosto de 2017) se menciona que en el 2017 "los ingresos por concepto de canon minero para los gobiernos regionales y locales ascendieron a $\mathrm{S} /$. 1862,7 millones a julio de este año" (párr. 1). Mientras que en el artículo de Gestión (13 de julio de 2018) publicado un año después se indica que

Las transferencias de recursos generados por la minería hacia las regiones en el 2018, por concepto de canon minero, ascendieron este año a S/ 3158 millones, monto que superó en $70 \%$ a lo registrado en 2017 (S/ 1863 millones), informó el Ministerio de Energía y Minas (MEM). Así mismo, indica que a nivel de regiones, la que tuvo mayor monto transferido fue Áncash con S/ 1085 millones (párr. 1).

En términos generales, se puede indicar que hay una tendencia al incremento en cuanto a la recaudación de este concepto, tanto a nivel nacional como regional, por ejemplo, Áncash en 2018 superó a sus dos años predecesores.

\section{Tiempos y costos de implementación}

A continuación, se detallan el tiempo y los costos invertidos en el momento "antes" y el momento "después". En la Tabla 6 se muestra un cuadro comparativo del tiempo:

En la tabla anterior se visualizan los tiempos de aplicación de los dos cuestionarios ("antes", aplicando el cuestionario original del SABP, y "después", aplicando el cuestionario propuesto), donde se observa que el cuestionario sugerido cuenta con una cantidad de ítems menor y se aplica en menos tiempo. Además, la fase de asistencia técnica ya no toma 8 sino 6 horas académicas y los días de viáticos ya no son 9 días sino solo 7 . Por otro lado, la fase de evaluación o monitoreo ya no es realizada en 6 horas académicas sino solo 4,5 horas, así como los días de viáticos se redujeron un día, pasando de 7 a 6 días.

Para los costos que se emplean en la aplicación del cuestionario inicial versus los que tomaría el cuestionario propuesto se ha desarrollado la Tabla 7:

En la Tabla 7 se aprecian dos fases (capacitación y asistencia técnica), las cuales pueden ser realizadas por un solo evaluador en una intervención, ello para evitar el pago por concepto de pasajes a un evaluador adicional que, dependiendo de la región a intervenir, podría ascender hasta S/900. Además de eso, con la aplicación del nuevo cuestionario, la fase de asistencia técnica ya no se estimaría en S/ 5620, sino en S/ 4260. También la fase de evaluación o monitoreo ya no ocasionaría un costo de S/ 4560, sino uno de S/ 3630. Para visualizar de manera comparativa los cambios en costeos, se presenta la Tabla 8.

Tabla 6. Tiempos de aplicación de actividades.

\begin{tabular}{|c|c|c|c|c|c|c|c|c|}
\hline 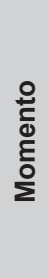 & $\begin{array}{l}\text { Nombre de } \\
\text { evaluador }\end{array}$ & Fase & 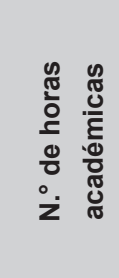 & 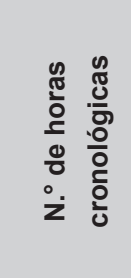 & 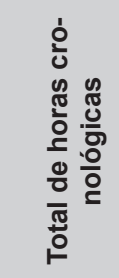 & 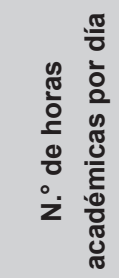 & 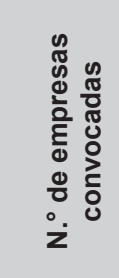 & 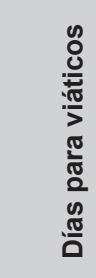 \\
\hline \multirow{3}{*}{ 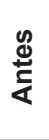 } & \multirow{2}{*}{ Evaluador 1} & Capacitación & 20 & 15 & 15 & 5 & 20 & 5 \\
\hline & & Asistencia técnica & 8 & 6 & 60 & 9 & 10 & 9 \\
\hline & Evaluador 2 & Evaluación o monitoreo & 6 & 4,5 & 45 & 9 & 10 & 7 \\
\hline \multirow{3}{*}{ 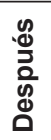 } & \multirow{2}{*}{ Evaluador 1} & Capacitación & 20 & 15 & 15 & 5 & 20 & 5 \\
\hline & & Asistencia técnica & 6 & 4,5 & 45 & 9 & 10 & 7 \\
\hline & Evaluador 2 & Evaluación o monitoreo & 4,5 & 3,375 & 33,75 & 8,44 & 10 & 6 \\
\hline
\end{tabular}

Fuente: Elaboración propia. 
Tabla 7. Costeo de aplicación de actividades.

\begin{tabular}{|c|c|c|c|c|c|c|c|c|c|c|c|}
\hline \multirow[t]{2}{*}{ 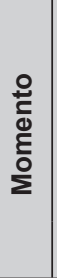 } & \multirow[t]{2}{*}{ 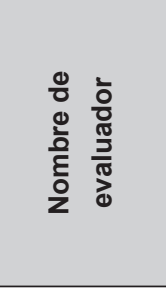 } & \multirow[t]{2}{*}{ Fase } & \multirow[t]{2}{*}{ 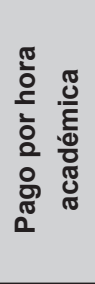 } & \multirow[t]{2}{*}{ 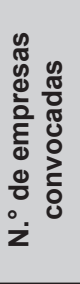 } & \multirow[t]{2}{*}{ 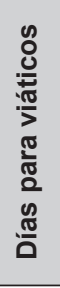 } & 总 & 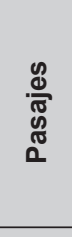 & $\begin{array}{l}\circ \frac{}{ㅎ} \\
\frac{\pi}{0} \\
\frac{0}{1} \\
\text { 오 }\end{array}$ & \multirow[t]{2}{*}{ 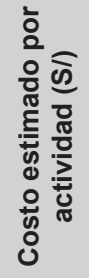 } & \multirow[t]{2}{*}{ 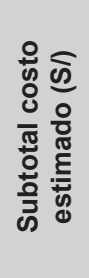 } & \multirow[t]{2}{*}{ 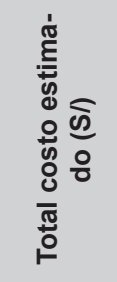 } \\
\hline & & & & & & ๘ & ๘ & ஸे & & & \\
\hline \multirow{3}{*}{ 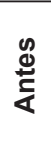 } & \multirow{2}{*}{ Evaluador 1} & Capacitación & 50 & 20 & 5 & 900 & 300 & 1000 & 2200 & \multirow{2}{*}{7820} & \multirow{3}{*}{12380,0} \\
\hline & & Asistencia técnica & 50 & 10 & 9 & 1620 & 0 & 4000 & 5620 & & \\
\hline & Evaluador 2 & Evaluación o monitoreo & 50 & 10 & 7 & 1260 & 300 & 3000 & 4560 & 4560 & \\
\hline \multirow{3}{*}{ 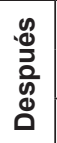 } & \multirow{2}{*}{ Evaluador 1} & Capacitación & 50 & 20 & 5 & 900 & 300 & 1000 & 2200 & \multirow{2}{*}{6460} & \multirow{3}{*}{10090,0} \\
\hline & & Asistencia técnica & 50 & 10 & 7 & 1260 & 0 & 3000 & 4260 & & \\
\hline & Evaluador 2 & Evaluación o monitoreo & 50 & 10 & 6 & 1080 & 300 & 2250 & 3630 & 3630 & \\
\hline
\end{tabular}

Fuente: Elaboración propia.

Tabla 8. Costeo comparativo de actividades o fases en el momento antes y el momento después

\begin{tabular}{|l|l|l|}
\hline & Actividades cuestionario SABP & Actividades cuestionario propuesto \\
\hline Costo nominal estimado (S/) & 12380,00 & 10090,00 \\
\hline Tasa promedio mensual inflación & $0,26 \%^{*}$ & $0,11 \%^{* *}$ \\
\hline Costo real estimado (S/) & 12347,81 & 10078,91 \\
\hline
\end{tabular}

Fuente: Elaboración propia.

* Tasa promedio mensual setiembre 2016

** Tasa promedio mensual 2017

Ratio $=($ Costo real estimado actividades cuestionario SABP - Costo real estimado cuestionario propuesto) / Costo real estimado cuestionario propuesto

$$
=(12347,81-10078,91) / 12347,81=0,18
$$

Ratio: $18 \%$

Por lo tanto, se puede concluir que la aplicación del nuevo cuestionario propuesto alcanzara una reducción en los costos del 18\%.

\section{Otros impactos}

Además de los beneficios previos señalados, este sistema también ayudará a combatir la informalidad, puesto que los requisitos principales para poder aplicar serán:

- Tener un negocio registrado en SUNAT, que cuente con RUC vigente y activo.

- Contar con la licencia de funcionamiento emitida por la municipalidad de la zona donde se ubique el establecimiento.

- Estar registrado o iniciar su registro en el órgano competente correspondiente a la zona (DIRCETUR, GERCETUR, DIRCETURA,
MINCETUR, etc. $)^{2}$, ostentando clase y/o categoría o siendo un establecimiento no clasificado/no categorizado.

Finalmente, este sistema y el cuestionario propuesto podrán aplicarse en cualquier destino que cuente con establecimientos de hospedaje tipo mypes; sin embargo, se recomienda que pueda ser aplicado desde la formación de un destino turístico, es decir en destinos emergentes.

\section{RECOMENDACIONES}

- El sistema aplicado puede extenderse a empresas en distintos lugares con potencial turístico, a fin de desarrollar el entorno emergente de la ubicación de los negocios, donde aún no se ha llegado a intervenir ni con este programa ni con algún otro.

- El cuestionario propuesto puede sistematizarse; además es posible que, a través de una plataforma online, se permita el

2 Dirección Regional de Comercio Exterior y Turismo (DIRCETUR), Gerencia Regional de Comercio Exterior y Turismo (GERCETUR), Dirección Regional de Comercio Exterior, Turismo y Artesanía (DIRCETURA), Ministerio de Comercio Exterior y Turismo del Perú (MINCETUR). 
llenado y revisión del cuestionario, ahorrando tiempo y dinero en recursos (humanos: evaluadores y equipo técnico; económicos: menor personal y equipo de cómputo a menor coste, entre otros).

- Este sistema y el cuestionario propuesto podrían extenderse a empresas de mayor envergadura como aquellas de 3 estrellas y hasta de mayor categoría como las de 4 estrellas en ciudades o destinos donde la actividad turística ya se viene desarrollando, pero aún no cuentan con el seguimiento de estándares de calidad. Esto sería un incentivo para poder crecer y elevar el cumplimiento exigido en el cuestionario, pasando del $80 \%$ del cumplimiento de nivel de calidad al $90 \%$ para este tipo de establecimientos.

\section{CONCLUSIONES}

1. Con los resultados obtenidos se logró cumplir el objetivo general de la presente investigación, el cual era implementar el Sistema de Aplicación de Buenas Prácticas, que permite mejorar la calidad de servicio ofrecido en las mypes de hospedaje de los destinos emergentes en el Perú. Por lo tanto, se demuestra la hipótesis alternativa: la adecuada gestión del proceso estratégico afecta la mejora de la calidad de servicio de las mypes de hospedaje.

2. Al ser el cuestionario existente, muy amplio y tedioso de aplicar, se propuso una reestructuración del mismo, incluyendo solo aquellos ítems relevantes para la obtención del puntaje final. El cuestionario inicial tenía 180 preguntas o ítems, mientras que, usando el análisis de correlación, se redujo a 138 preguntas, dentro de las cuales se incluyeron también algunos aspectos adicionales relevantes.

3. Dentro de los cuatro factores o procesos englobados en el cuestionario SABP: proceso estratégico, proceso principal, proceso de soporte y competencias de los colaboradores; se incluyen ciertos aspectos con los que debe cumplir un establecimiento de hospedaje. Sin embargo, luego de un análisis de correlación, se concluye que no todos esos factores son pertinentes para la obtención de un alto nivel de calidad. Para ello, el análisis de correlación y un análisis específico ayudaron a identificar que el proceso de soporte es un aspecto irrelevante. Así, se concluyó en solo considerar tres de los cuatro procesos, los cuales serían suficientes para alcanzar la mejora de la calidad de servicios.
4. El impacto del proyecto, si se desea aplicar a la región Ica, ocasiona una reducción aproximada del $18 \%$ del costo.

5. Más de quince regiones del país reciben transferencias por concepto de canon minero; sin embargo, los gobiernos locales no siempre cuentan con proyectos de mejora a la localidad relacionados con la actividad turística. Ese capital destinado podría invertirse en dichos proyectos y contar con programas que permitan a los habitantes de estas comunidades mejorar su calidad de vida basada en el turismo y en actividades complementarias.

6. Si este sistema quisiera aplicarse a otras realidades, se podrá realizar de manera correcta asegurando que el entorno de aplicación sea similar al de la región Ica o, en general, a una empresa bajo el régimen del Reglamento de establecimientos de hospedaje del Perú.

7. El cuestionario propuesto podría adaptarse a otros rubros como el hospitalario, donde se requiere de una atención de calidad en diferentes aspectos, y cumplir todos los requerimientos de buenas prácticas mediante los diferentes elementos que se manejan en este tipo de actividad.

\section{AGRADECIMIENTO}

Al Mg. Alejandro Gallegos Chocce por su asesoramiento continuo y desinteresado, compartiendo y aportando enormemente en esta investigación con sus conocimientos, experiencias y profesionalidad.

A las diferentes entidades y empresarios que hicieron posible que esta investigación se desarrolle satisfactoriamente.

\section{REFERENCIAS BIBLIOGRÁFICAS}

[1] Barbero, I. (2007). Gestión de la calidad en las organizaciones no lucrativas de intervención social. Recuperado de http:// www.fundacionede.org/calidad/master/es/libro/ Gesti\%C3\%B3n\%20de\%20la\%20calidad\%20 en $\% 20$ las $\% 20$ organizaciones $\% 20$ no $\% 20$ lucrativas $\% 20$ de $\% 20$ intervenci\%C3\%B3n\%20 social.pdf

[2] Cabeza, M. (2001). La logística en la actividad turística. Revista Venezolana de Análisis de Coyuntura, 7(2), 257-264.

[3] ConexiónESAN(25demayode2016). Marketing de servicios: significado y características. 
Conexión ESAN. Recuperado de https://www. esan.edu.pe/apuntes-empresariales/2016/05/ marketing-servicios-significado-caracteristicas/

[4] Cubillos, M. y Rozo, D. (2009). El concepto de calidad: Historia, evolución e importancia para la competitividad. Revista de la Universidad de La Salle, (48), 80-99.

[5] Decreto Supremo N. ${ }^{\circ} 001$ (9 de junio de 2015). Reglamento de establecimientos de hospedaje. El Peruano, Normas Legales: 554648.

[6] Francisco, C. de (2014). Tendencias turísticas en el mercado español debidas a segmentos emergentes y potenciales (Tesis de pregrado). Universidad de Valladolid, Segovia.

[7] Gestión (1 de agosto de 2017). Ingresos por canon minero en los gobiernos regionales y locales aumentaron $24.4 \%$ a julio. Gestión. Recuperado de https://gestion.pe/economia/ ingresos-canon-minero-gobiernos-regionaleslocales-aumentaron-24-4-julio-140691

[8] Gestión (13 de julio de 2018). MEM: Canon minero de 2018 fue $70 \%$ superior al del año pasado. Gestión. Recuperado de https:// gestion.pe/economia/mem-canon-minero2018-70-superior-ano-pasado-238429

[9] López, E., Cabrera, Y., López, E. y Puerto, A. (2018). Calidad percibida en servicios de asistencia al adulto mayor. Medisur, 16(3), 437-463.

[10] Ministerio de Comercio Exterior y Turismo (MINCETUR) (2016). Plan Estratégico Nacional de Turismo 2025. Recuperado de https:// www.mincetur.gob.pe/wp-content/uploads/ documentos/turismo/documentos/PENTUR/ PENTUR_Final_JULIO2016.pdf

[11] Ministerio de Comercio Exterior y Turismo (MINCETUR) (2017). Plan Nacional de Calidad Turística. Recuperado de https://www.mincetur. gob.pe/wp-content/uploads/documentos/ turismo/CALTUR/pdfs_documentos_Caltur/ CALTUR_2017_2025.pdf

[12] Organización de las Naciones Unidas para la Alimentación y la Agricultura (FAO) (julio de 2015). Plantilla de buenas prácticas. Recuperado de http://www.fao.org/3/a-as547s. pdf

[13] Organización de las Naciones Unidas para la Educación, la Ciencia y la Cultura (UNESCO) (2017). About the MOST Programme. Recuperado de http://www.unesco.org/new/ en/social-and-human-sciences/themes/mostprogramme/about-most/

[14] Organización Mundial del Turismo (OMT) (2002). Turismo: Panorama 2020. Previsiones mundiales y perfiles de los segmentos de mercado. Madrid, España: Organización Mundial del Turismo.

[15] Sanabria, P., Romero, V. y Flórez C. (2014). El concepto de calidad en las organizaciones: una aproximación desde la complejidad. Universidad \& Empresa, 16(27), 165-213. Recuperado de http://www.redalyc.org/ pdf/1872/187241606007.pdf 


\section{Improving service quality offered by lodging industry SMEs located in emerging Peruvian destinations using the Application of Good Practices System}

SUSAN HeRrera MARÍN

Received: 08/11/2018 Accepted: 07/01/2019

\begin{abstract}
This paper is the result of the need for SMEs in the lodging industry to improve service quality while remaining cost-effective. The Sistema de Aplicación de Buenas Prácticas [Application of Good Practices System] (SABP) is a tool for SMEs, as it improves quality of services offered and their positioning, leveraging the zone and destination location, in order to optimize the quality of life in the community. Thus, this study proposes an application methodology that adjusts to the progress of said businesses, so that a balance between adequate administrative management processes, service, infrastructure and equipment, collaborator performance, and support areas is found
\end{abstract}

Keywords: Good practices; quality of service; MSE; accommodation; destination.

\section{INTRODUCTION}

Tourism is one of the most important sources for the development of cities around the world. In this respect, Peru has become a relevant destination, since it has those resources that can be exploited wisely and sustainably. In addition, tourism can provide satisfaction to both small business owners and all other participants who are involved directly or indirectly with the performance of this economic activity. Thus, on this subject, Cabeza (2001) notes that "los expertos coinciden en que el turismo, conjuntamente con las comunicaciones y la informática, serán los tres sectores que regirán la economía mundial del tercer milenio, caracterizado por una penetración de la tecnología en todos los aspectos de la vida [experts agree that tourism, together with communications and information technology, will be the three sectors that will govern the world economy in the third millennium, characterized by the introduction of technology in all aspects of life] (p. 257). Additionally, the World Tourism Organization (UNWTO) states in its Tourism 2020 Vision (2002) report that revenues from international tourism (excluding transport) are expected to reach 2 trillion dollars by 2020 and that more than 5000 million dollars will be spent every day, while the number of international tourist arrivals worldwide will increase to almost 1600 million in 2020 . Likewise, Francisco (2014) says that "las principales zonas de crecimiento, en lo que a inversiones extranjeras se refiere, se están trasladando a China, países latinoamericanos seguros y emergentes $y$, por último, a países africanos con las mismas condiciones" [the main growth areas, in regards to foreign investments, are moving to China, safe and emerging Latin American countries and African countries under the same conditions] ( $p$. 30)

This study focuses on one of the main problems that tourism entails, which arises with the appearance of tourism service providers in emerging destinations in Peru, as they do not always operate a quality product or service. Therefore, they require spe-

1 Licenciada* in Tourism Administration, with a master's degree in Operations Management and Logistics Services from the Universidad Nacional Mayor de San Marcos. Experienced in quality of tourism and hotel services and teaching. Currently working as an assistant to the tourism and hospitality job board of Universidad San Martín de Porres. Lima, Peru. E-mail: susan.herrera@unmsm.edu.pe

* In Peru, Licenciatura or Título is a professional title granted after having defended a thesis or passing a course in accordance with the university's regulations once the undergraduate program has been completed. 
cial attention and they need to have these systems adapted to their micro and small enterprises in this type of destination, improving their knowledge of quality guidelines for these areas.

The use of the preexisting Application of Good Practices System (SABP) is proposed for MSEs in the lodging sector and as a measuring tool which has been redesigned in order to be an easy-to-use tool that is more in line with the current reality of destinations that have either not developed or have only incipient tourist activity. It is based on the National Strategic Plan for Tourism of Peru (PENTUR), which states, regarding Peruvian tourism in the future, that it is "elaborado con metas hacia el 2025, que cuenta con un modelo de desarrollo turístico, siendo uno de sus 4 pilares la diversificación y consolidación de la oferta" [designed with goals towards 2025, that incorporates a model of tourism development, being one of its 4 pillars the diversification and consolidation of the provision of the offering] (Ministerio de Comercio Exterior y Turismo, 2016, para. 6)

From the above, it can be specified that the purpose of this proposal is to provide entrepreneurs, local governments, and regional governments with a tool to improve the quality of service in lodging establishments. The goal is to take into account the "before" and "during" of the planning and operation of an establishment of this type and to be able to ensure the execution of quality services generating a greater flow of both domestic and international visitors and, at the same time, have a positive impact on the vicinities surrounding these businesses.

\section{Background}

Quality has been a predominant feature of mankind's throughout the evolutionary process, always searching for the greatest benefit with the least amount of effort. By 1920, quality began to make more substantial changes, which are summarized below in Table 1.

"Un avance significativo, dentro del concepto de calidad, es el hecho de incorporar al cliente como un actor fundamental [...]. La calidad no es un resultado final sino [...] un camino sin final, un sendero que siempre es necesario recorrer." [A significant advance, within the concept of quality, is the act of incorporating the client as a fundamental actor [...]. Quality is not a final result but [...] an endless path that is always necessary to travel] (López et al., 2018, p. 444). In this way, its management assumes quality as a means to achieve goals or as an organizational horizon that guides action, recognizing both predictable as well as random, unpredictable and unexpected elements (Sanabria, Romero \& Flórez, 2014). Quality is an endless reality, since it is a path where we must always travel to achieve the goals set around the progress of SMEs, the client and its fullness. In this regard, the Food and Agriculture Organization of the United Nations (2015) points out that "a good practice is not only a practice that is good, but a practice that has been proven to work well and produce good results, and is therefore recommended as a model" (p.1). On the other hand, the United Nations Educational, Scientific and Cultural Organization (2017), as part of its Manage-

Table 1. Evolution of the concept of quality.

\begin{tabular}{|c|c|c|c|}
\hline Stage & Concept & Purpose & Historical context \\
\hline $\begin{array}{c}\text { Quality control via } \\
\text { inspection }\end{array}$ & $\begin{array}{c}\text { Use of quality control techniques by } \\
\text { inspection and statistical methods } \\
\text { that allow the identification of defec- } \\
\text { tive products. }\end{array}$ & $\begin{array}{c}\text { Satisfy standards and technical conditions } \\
\text { of a product. }\end{array}$ & $\begin{array}{c}\text { End of the First World } \\
\text { War }\end{array}$ \\
\hline Quality assurance & $\begin{array}{c}\text { Quality is assured without cost } \\
\text { sparing but guaranteeing greater } \\
\text { volume of production in less time. }\end{array}$ & $\begin{array}{c}\text { Guarantee an effective product in reques- } \\
\text { ted quantities and timeframes. }\end{array}$ & World War II \\
\hline Total quality & $\begin{array}{c}\text { Use of systems and procedures to } \\
\text { avoid defective products in all func- } \\
\text { tional areas of the organization. }\end{array}$ & $\begin{array}{c}\text { Satisfy the client by avoiding mistakes. } \\
\text { Generate competitiveness with the lowest } \\
\text { cost and highest employee participation. }\end{array}$ & 70 and 90s \\
\hline $\begin{array}{c}\text { Continuous impro- } \\
\text { vement }\end{array}$ & $\begin{array}{c}\text { Quality leaders are trained to em- } \\
\text { power processes. }\end{array}$ & $\begin{array}{c}\text { Satisfy the client by avoiding mistakes. } \\
\text { Generate competitiveness by progressively } \\
\text { reducing costs and increasing profits. } \\
\text { Rely on continuous improvement teams }\end{array}$ & 90s to present day \\
\hline
\end{tabular}

Source: Prepared by the author based on the work of Cubillos \& Rozo (2009) 
ment of Social Transformations (MOST) program, specifies that good practices should be innovative, effective, sustainable and replicable, since they are now necessary resources.

Also, we can call "sistemas a todas aquellas referencias, como códigos, normas o modelos, que proporcionan directrices, orientaciones o requerimientos referentes al modo cómo ha de gestionarse una organización y/o prestarse un servicio" [all those references, such as codes, standards or models, which provide guidelines, directions or requirements regarding how an organization should be managed and/or how a service should be provided, systems] (Barbero, 2007, p. 5). In an article entitled Marketing de servicios: significado y características (Marketing of services: meaning and characteristics), Conexión ESAN (May 25, 2016) mentions that the main characteristics of services are intangibility, heterogeneity/ variability, perishability and the inseparability/simultaneity of production and consumption.

\section{Application of Good Practices System (SABP)}

The Ministry of Foreign Trade and Tourism of Peru (MINCETUR) (2017) indicates that

El Plan Nacional de Calidad Turística del Perú (CALTUR) es un instrumento de gestión del Sector Turismo que establece las estrategias y líneas de acción en materia de calidad, para posicionar al Perú como un destino global de calidad, logrando que sus destinos y productos sean percibidos como espacios y experiencias de alto valor en todos sus componentes [The Plan Nacional de Calidad Turística del Perú (National Tourism Quality Plan of Peru) (CALTUR) is a management tool for the Tourism Sector that establishes the strategies and lines of action in terms of quality to position Peru as a global quality destination, making its destinations and products perceived as spaces and experiences of high value in all its components] (para. 1).

The SABP is the CALTUR tool that allows the inclusion of tourism service providers in continuous improvement processes and is applied by the Centro de Formación en Turismo [Tourism Training Center] (CENFOTUR). In addition, it is mainly aimed at lodging, restaurant and related services, travel and tourism agencies.

In accordance with Supreme Decree No. 001-2015-MINCETUR of the Reglamento de establecimientos de hospedaje (Regulations of lodging establishments) (June 9, 2015), these are classified as: hotel, 1 to 5 stars; apart-hotel, 3 to 5 stars; hostal, 1 to 3 stars; and albergue, without category (art. 3). In addition, it is important to define the four verification aspects that the instrument has developed to measure the quality of the lodging establishments according to the SABP, which is the purpose for this study. The questionnaire for lodging establishments (SABP evaluation phase) is shown below:

Strategic Process. Established by strategy, management and back office areas. It is divided into administration, marketing and sales, and logistics.

Main process. Consisting of four pillars of the operation: reception and reservations (infrastructure and equipment), reception and reservations (service management), housekeeping (infrastructure and equipment) and housekeeping (service management).

Support process. Consists of food and beverage, maintenance, and security.

Collaborator Competencies. Comprising one of the most important aspects of the organization.

\section{METHODOLOGY}

The sample is determined by thirteen lodging establishments in the Ica Region. The procedure used to select the sample was the probabilistic method and simple random sampling. In addition, the data collection techniques used were:

Interviews and surveys prepared for microentrepreneurs from the hotel sector in the locality of Ica (SABP questionnaire and proposed questionnaire).

Research work, review of the various historical company data related to the research variables.

Analysis of quantitative content carried out using the statistical analysis software Minitab 17.

\section{RESULTS}

\section{Reformulation and creation of the new proposed questionnaire}

The development of the new questionnaire was based on the "before" moment sample using Minitab 17 to progressively identify the least relevant items and evaluate the quality of the establishment. In this way, it was achieved by comparing the total result or quality level achieved by the company versus each subtotal result or score achieved in each part contained in said questionnaire and these in 
turn with their subparts. The number of items contained in the initial questionnaire up to the proposed questionnaire is shown in Table 2.

As shown in the previous table, the initial questionnaire or Asys had 180 items, while the To be questionnaire had 131 after applying Minitab 17 to the samples scores percentages taken and discarding those items based on the prior knowledge of the author. Likewise, in the To be improved version, 7 new aspects to be evaluated have been included or modified, resulting in the new proposed questionnaire with a total of 138 items.

\section{Survey application results}

The survey application results at the "2016 (before)" moment, where the Asys questionnaire was used, and at the "2017 (after)" moment, in which the proposed questionnaire was used - are shown in Table 3.

In this way, it is observed that company scores have improved between the two interventions, which

Table 2. Quality criteria in questionnaires.

\begin{tabular}{|c|c|c|c|c|}
\hline \multirow{2}{*}{ Dimensions } & \multirow{2}{*}{ Subdimensions } & \multicolumn{3}{|c|}{ Criteria } \\
\hline & & Asys & To be & To be improved \\
\hline \multirow[t]{3}{*}{ Management of the strategic process } & Administration & 12 & 8 & 0 \\
\hline & Marketing and sales & 6 & 6 & 0 \\
\hline & Logistics & 7 & 5 & 0 \\
\hline \multirow[t]{4}{*}{ Management of the main process } & $\begin{array}{l}\text { Reception and reservations (infrastructure and } \\
\text { equipment) }\end{array}$ & 18 & 15 & 2 \\
\hline & Reception and reservations (service management) & 28 & 26 & 1 \\
\hline & Housekeeping (infrastructure and equipment) & 63 & 46 & 0 \\
\hline & Housekeeping (service management) & 20 & 17 & 0 \\
\hline \multirow[t]{3}{*}{ Management of the support process } & Food and beverage & 9 & 1 & 2 \\
\hline & Maintenance & 2 & 0 & 1 \\
\hline & Security & 8 & 1 & 1 \\
\hline Management of collaborator competencies & Collaborators & 7 & 6 & 0 \\
\hline \multicolumn{2}{|l|}{ TOTAL } & 180 & 131 & 7 \\
\hline
\end{tabular}

Source: Prepared by the author.

Table 3. Results of the application of questionnaires.

\begin{tabular}{|c|l|c|c|c|c|}
\cline { 3 - 6 } \multicolumn{2}{c|}{} & \multicolumn{2}{c|}{ 2016 (before) } & \multicolumn{2}{c|}{ 2017 (after) } \\
\hline N. & \multicolumn{1}{c|}{ Establishment } & Score obtained & Qualification & Score obtained & Qualification \\
\hline 1 & Hotel Suite Grau & $70.07 \%$ & Improving & $76.69 \%$ & Improving \\
\hline 2 & Hotel Duna Dorada & $81.91 \%$ & Acceptable & $89.19 \%$ & Acceptable \\
\hline 3 & Hostal Lindo Perú & $66.69 \%$ & Improving & $74.25 \%$ & Improving \\
\hline 4 & Hostal Antonio's & $66.03 \%$ & Improving & $73.12 \%$ & Improving \\
\hline 5 & Hotel Continental's & $60.29 \%$ & Improving & $69.36 \%$ & Improving \\
\hline 6 & Hostal Evelyn & $73.90 \%$ & Improving & $76.03 \%$ & Improving \\
\hline 7 & Hotel El Parral Inn & $78.31 \%$ & Improving & $80.26 \%$ & Acceptable \\
\hline 8 & Hostal Oasis & $75.07 \%$ & Improving & $79.89 \%$ & Acceptable \\
\hline 9 & Hostal Monarca & $77.35 \%$ & Improving & $78.01 \%$ & Improving \\
\hline 10 & Hostal Campo Alegre & $74.41 \%$ & Improving & $77.16 \%$ & Improving \\
\hline 11 & Hostal Vega's & $65.59 \%$ & Improving & $78.29 \%$ & Improving \\
\hline 12 & Hotel El Embrujo & $80.44 \%$ & Acceptable & $81.86 \%$ & Acceptable \\
\hline 13 & Hostal El Médano & $79.04 \%$ & Improving & $79.61 \%$ & Acceptable \\
\hline & Averages & $73.01 \%$ & & $77.98 \%$ & \\
\hline
\end{tabular}

Source: Prepared by the author. 
demonstrates that the new implemented questionnaire obtained good results and met the planned research objective.

\section{Hypothesis testing}

\subsection{General hypothesis}

Initially a normality analysis was carried out via the Anderson-Darling test, which is the most effective in detecting non-normality in the distribution tails, since the latter are the most critical within the distribution. Likewise, it is important to mention that it is relying upon a $95 \%$ confidence interval to conclude that the data follow a normal distribution. The main figures obtained from this test for each moment (before and after) are shown in Table 4.

Table 4. Results of normality analysis.

\begin{tabular}{|l|c|c|}
\hline & Before & After \\
\hline A-Squared & 0.35 & 0.19 \\
\hline P-Value & 0.425 & 0.885 \\
\hline Media & 0.73008 & 0.77975 \\
\hline
\end{tabular}

Source: Prepared by the author.

It is shown that the distribution of the quality data in both application moments has a normal behavior (P-Value $>0.05)$. In the same way, it was determined that the independent variable "Application of Good Practices System" is discrete and the dependent variable "Improvement of the quality of service of lodging MSEs" is continuous, so the Two-Sample T-Test must be performed using Minitab 17. After the Two-Sample T-Test was analyzed, it was concluded that the implementation of the independent variable affected the dependent variable because the $P$-Value $<0.05$, due to which the alternative hypothesis is demonstrated.

\subsection{Specific hypothesis}

It was determined that the dimensions of the independent variable (the management of the strategic process, the management of the main process, the management of the support process and the management of collaborators competencies) are continuous. In the same way, it was found that the dependent variable "Improvement of the quality of service of lodging SMEs" is continuous, for which correlation and regression tests were carried out using Minitab 17.

Correlation and regression analysis. This analysis serves to measure the strength and direction of the association between two continuous variables. In this case, Pearson's correlation coefficient was used, while the regression analysis was used to study the relationship between variables or quantitative factors referred to the same group of units observed. The main data of this test applied for each of the specific hypotheses are shown in Table 5.

From Table 5 it is concluded that the management of the strategic process is significant and affects service quality "before" by $53.78 \%$. Likewise, there is a strong relationship between them; the management of the main process is significant and affects service quality "before" by $97.89 \%$. Likewise, there is also a strong relationship between them; the management of the support process is not significant and does not affect service quality "before", there is no relationship between them. The management of the collaborators competencies is significant and affects service quality "before" by $43.78 \%$, and therefore there is a high/medium average relationship between them.

\section{Quality summary report at the "before" moment}

Correlation and regression analysis. The correlation analysis to the four dimensions was performed with respect to service quality "before", in which it is observed that the dimension management of the support process has a Pearson's coefficient close to 0 and P-Value> 0.05 (equal to 0.999), which means that this dimension has no relation to the problem.

In addition, after the regression analysis was applied to the remaining three dimensions (management of the strategic process, management of the main process and management of collaborator competencies) versus the quality of the service "before", it is concluded that only those three dimensions are significant and they affect together the quality of

Table 5. Results of correlation and regression analysis.

\begin{tabular}{|c|c|c|c|c|c|}
\hline & & Hypothesis 1 & Hypothesis 2 & Hypothesis 3 & Hypothesis 4 \\
\hline \multirow{2}{*}{ Correlation } & Pearson's coefficient & 0.733 & 0.989 & 0 & 0.662 \\
\cline { 2 - 6 } & P-Value & 0.004 & 0.000 & 0.999 & 0.014 \\
\hline Regression & R-Squared & $53.78 \%$ & $97.89 \%$ & $0 \%$ & $43.78 \%$ \\
\hline
\end{tabular}

Source: Prepared by the author. 
service by $99.53 \%$, because the P-Value $<0.05$ and the $\mathrm{R}^{2}$ is $99.53 \%$. Therefore, the dimension management of the support process is discarded.

Therefore, the equation that represents the improvement of the quality of service of the lodging MSEs, based on these three dimensions, is the following:

$$
\begin{aligned}
\text { QUALITY }= & 0.0302+0.1860 \text { I. }- \text { Strategic process }+0.6333 \\
& \text { II. - Main process }+0.1222 \text { IV. }- \text { Collaborator com- } \\
& \text { petencies }
\end{aligned}
$$

\section{IMPACTS}

\section{Proposed problem solution}

The suggested solution is the application of the system using the new proposed questionnaire, which will make possible service quality improvement for lodging industry SMEs in emerging destinations in Peru, using fewer time and money resources, which will optimize the system application. Also, the implementation of this system can be financed by regional or local governments, such as the more than 15 main regions that receive transfers from Peru's mining tax. In an article in Gestión (August 1, 2017) it is mentioned that in 2017 "los ingresos por concepto de canon minero para los gobiernos regionales $y$ locales ascendieron a S/. 1862,7 millones a julio de este año" [income from Peru's mining tax for regional and local governments rose to S/. 1862.7 million as of July this year] (para. 1). While in another article Gestión (July 13, 2018) published a year later it is indicated that.

Las transferencias de recursos generados por la minería hacia las regiones en el 2018, por concepto de canon minero, ascendieron este año a S/ 3158 millones, monto que superó en $70 \%$ a lo registrado en 2017
(S/ 1863 millones), informó el Ministerio de Energía y Minas (MEM). Así mismo, indica que a nivel de regiones, la que tuvo mayor monto transferido fue Áncash con S/ 1085 millones. [The transfers of resources generated by mining to the regions in 2018, per Peru's mining tax, rose this year to $\mathrm{S} /$ 3158 million, an amount that exceeded $70 \%$ of what was registered in 2017 (S/ 1863 million), according to the Ministry of Energy and Mines (MEM). Likewise, it indicates that at the regional level, the region with the highest amount transferred was Áncash with S/. 1085 million] (paragraph 1).

In general terms, it can be said that there is a tendency to increase in terms of collection, both nationally and regionally, for example, Áncash in 2018 exceeded its two preceding years.

\section{Implementation times and costs}

Next, the time and costs invested in the "before" moment and the "after" moment are detailed. A comparative table of time is shown in Table 6.

The application times of the two questionnaires are shown in the previous table ("before", applying the original SABP questionnaire, and "after", applying the proposed questionnaire), where it is observed that the suggested questionnaire has a minor number of items and is applied in less time. In addition, the technical assistance phase no longer takes 8 but 6 academic hours and travel expenses are no longer for 9 days but only 7 . On the other hand, the evaluation or monitoring phase is no longer carried out in 6 academic hours but only in 4.5 hours; travel expensed days were reduced by one day, going

\begin{tabular}{|c|c|c|c|c|c|c|c|c|}
\hline 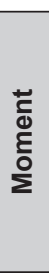 & $\begin{array}{l}\text { Name of } \\
\text { evaluator }\end{array}$ & Phase & 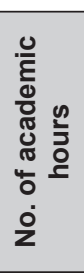 & 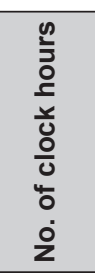 & 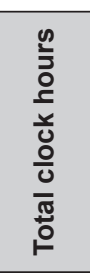 & 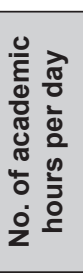 & 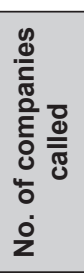 & 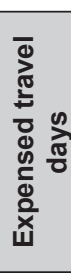 \\
\hline \multirow{3}{*}{$\begin{array}{l}0 \\
\frac{0}{4} \\
\text { Ф் }\end{array}$} & \multirow{2}{*}{ Evaluator 1} & Training & 20 & 15 & 15 & 5 & 20 & 5 \\
\hline & & Technical assistance & 8 & 6 & 60 & 9 & 10 & 9 \\
\hline & Evaluator 2 & Evaluation or monitoring & 6 & 4.5 & 45 & 9 & 10 & 7 \\
\hline \multirow{3}{*}{$\frac{\grave{\Phi}}{\frac{\Phi}{<}}$} & \multirow{2}{*}{ Evaluator 1} & Training & 20 & 15 & 15 & 5 & 20 & 5 \\
\hline & & Technical assistance & 6 & 4.5 & 45 & 9 & 10 & 7 \\
\hline & Evaluator 2 & Evaluation or monitoring & 4.5 & 3.375 & 33.75 & 8.44 & 10 & 6 \\
\hline
\end{tabular}
from 7 to 6 days.

Table 6. Times of activities application.

Source: Prepared by the author. 
Table 7. Activities application costing.

\begin{tabular}{|c|c|c|c|c|c|c|c|c|c|c|c|}
\hline \multirow[t]{2}{*}{ 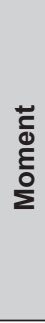 } & \multirow[t]{2}{*}{ 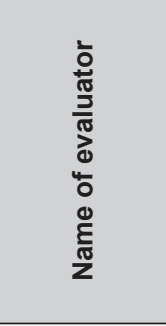 } & \multirow[t]{2}{*}{ Phase } & \multirow[t]{2}{*}{ 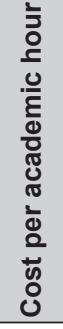 } & \multirow[t]{2}{*}{ 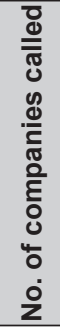 } & \multirow[t]{2}{*}{ 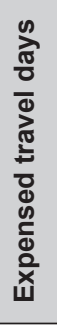 } & 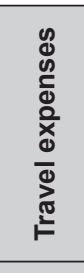 & $\begin{array}{l}\frac{n}{0} \\
\stackrel{0}{0} \\
i=\end{array}$ & 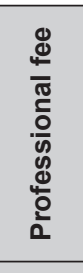 & \multirow[t]{2}{*}{ 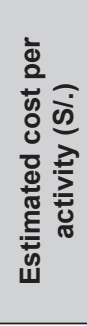 } & \multirow[t]{2}{*}{ 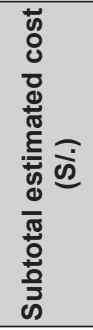 } & \multirow[t]{2}{*}{ 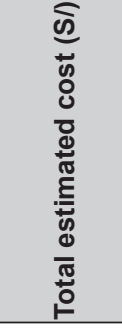 } \\
\hline & & & & & & $\dot{\omega}$ & $\dot{\omega}$ & $\dot{\omega}$ & & & \\
\hline \multirow{3}{*}{ 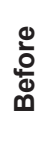 } & \multirow{2}{*}{ Evaluator 1} & Training & 50 & 20 & 5 & 900 & 300 & 1000 & 2200 & \multirow{2}{*}{7820} & \multirow{3}{*}{12380.00} \\
\hline & & Technical assistance & 50 & 10 & 9 & 1620 & 0 & 4000 & 5620 & & \\
\hline & Evaluator 2 & Evaluation or monitoring & 50 & 10 & 7 & 1260 & 300 & 3000 & 4560 & 4560 & \\
\hline \multirow{3}{*}{$\frac{\bar{\Phi}}{\frac{\mathbb{Q}}{\alpha}}$} & \multirow{2}{*}{ Evaluator 1} & Training & 50 & 20 & 5 & 900 & 300 & 1000 & 2200 & \multirow{2}{*}{6460} & \multirow{3}{*}{10090.00} \\
\hline & & Technical assistance & 50 & 10 & 7 & 1260 & 0 & 3000 & 4260 & & \\
\hline & Evaluator 2 & Evaluation or monitoring & 50 & 10 & 6 & 1080 & 300 & 2250 & 3630 & 3630 & \\
\hline
\end{tabular}

Source: Prepared by the author.

Table 8. Comparative costing of activities or phases in the moment before and the moment after

\begin{tabular}{|l|l|l|}
\hline & \multicolumn{1}{|c|}{ SABP questionnaire Activities } & Proposed questionnaire Activities \\
\hline Estimated nominal cost (S/.) & 12380.00 & 10090.00 \\
\hline Average monthly inflation rate & $0.26 \%^{*}$ & $0.11 \%^{* *}$ \\
\hline Estimated real cost $\mathbf{( S / . )}$ & 12347.81 & 10078.91 \\
\hline
\end{tabular}

Source: Prepared by the author.

${ }^{*}$ Average monthly rate on September 2016

** Average monthly rate in 2017

Table 7 has been developed for the costs used in the initial questionnaire application versus those of the proposed questionnaire:

Table 7 shows two phases (training and technical assistance), which can be carried out by a single evaluator during one visit to avoid paying for tickets for an additional evaluator that, depending on the region to be involved, could cost up to S/. 900 . In addition, with the application of the new questionnaire, the technical assistance phase would no longer be estimated at S/. 5 620, but at S/. 4260 . Also, the evaluation or monitoring phase would no longer result in a cost of S/. 4560 , but rather S/. 3 630 . Table 8 is presented to comparatively visualize the changes in cost:

Ratio $=($ Estimated real cost of SABP questionnaire activities - Estimated real cost of the proposed questionnaire) / Estimated real cost of the proposed questionnaire

$$
=(12347.81-10078.91) / 12347.81=0.18
$$

Ratio: $18 \%$
Therefore, it can be concluded that the application of the proposed new questionnaire will achieve a cost reduction of $18 \%$.

\section{Other impacts}

In addition to the above-mentioned benefits, this system will also help combat informality, since the main requirements to apply will be:

- Have a business registered in the National Superintendency of Tax Administration (SUNAT), with a current and active Taxpayer identification number (RUC).

- Possess an operating license issued by the municipality of the area where the establishment is located.

- Be registered or start registration in the competent body corresponding to the area (DIRCETUR, GERCETUR, DIRCETURA, MINCETUR, etc. $)^{2}$, showing class and/or category or being grouped as an unclassified/ uncategorized establishment. 
Finally, this system and the proposed questionnaire may be applied in any destination that has SME lodging facilities; however, it is recommended that it be applied during the formation of a tourist destination, that is to say in emerging destinations.

\section{RECOMMENDATIONS}

- The applied system can be extended to companies in different places with tourism potential in order to develop the emerging environment of the businesses location, where neither this nor any other application has been applied. The proposed questionnaire can be systematized; it is also possible that, through an online platform, it is possible to answer and revise the questionnaire, saving time and money resources (human: evaluators and technical team, economic: less staff and computer equipment at lower cost, among others).

- This system and the proposed questionnaire could be extended to larger companies such as those of 3 stars and even a higher category such as 4 stars in cities or destinations where tourism activity has already been developed, but still do not have quality standards guidelines. This would be an incentive to grow and raise the compliance required in the questionnaire, going from $80 \%$ of quality level compliance to $90 \%$ for this type of establishments.

\section{CONCLUSIONS}

1. With the results obtained, the general objective of this research was achieved, which was to implement the Good Practices Application System, which allows for the improvement of service quality offered by lodging industry SMEs in emerging destinations in Peru. Therefore, the alternative hypothesis is demonstrated: the adequate management of the strategic process affects the improvement of service quality in lodging industry SMEs.

2. As the existing questionnaire very broad and tedious to apply, a restructuring was proposed, including only those items relevant to obtain the final score. The initial questionnaire had 180 questions or items, while, using the correlation analysis, it was reduced to 138 questions, within which some additional relevant aspects were also included.
3. Within the four factors or processes included in the SABP questionnaire-strategic process, main process, support process and employee competencies-certain aspects that a lodging establishment must comply with were included. However, after a correlation analysis, it is concluded that not all of these factors are relevant to obtain a high-quality level. For this, the correlation analysis and a specific analysis helped to identify that the support process is an irrelevant aspect. Thus, it was concluded to only consider three of the four processes, which would be sufficient to achieve the improvement of the quality of services.

4. The impact of the project, if it is desired to apply to the Ica region, results in an approximate cost reduction of $18 \%$.

5. More than fifteen regions of the country receive transfers from Peru's mining tax; however, local governments do not always have local improvement projects related to tourism. This capital could be invested in these projects and have programs that allow the inhabitants of these communities to improve their quality of life based on tourism and complementary activities.

6. If this system was intended to other realities, it can be carried out in a correct manner, ensuring that the application environment is similar to that of the Ica region or, in general, to a company under the regulation of lodging establishments in Peru.

7. The proposed questionnaire could be adapted to other areas such as the hospitality sector, where different aspects of quality care is required, as is the completion of all good practices requirements through the different practices administered in this type of activity.

\section{ACKNOWLEDGEMENTS}

To Alejandro Gallegos Chocce, MBA, for his continuous and selfless advice, sharing and contributing enormously to this study with his knowledge, experience and professionalism.

To the different entities and business leaders who made this study possible.

\section{REFERENCES}

[1] Barbero, I. (2007). Gestión de la calidad en las organizaciones no lucrativas de intervención social. Retrieved from http://www. 
fundacionede.org/calidad/master/es/libro/ Gesti\%C3\%B3n\%20de\%20la\%20calidad\%20 en $\% 201$ as $\% 20$ organizaciones $\% 20$ no $\% 20$ lucrativas\%20de\%20intervenci\%C3\%B3n\%20 social.pdf

[2] Cabeza, M. (2001). La logística en la actividad turística. Revista Venezolana de Análisis de Coyuntura, 7(2), 257-264.

[3] Conexión ESAN (2016, May 25). Marketing de servicios: significado y características. Conexión ESAN. Retrieved from https://www. esan.edu.pe/apuntes-empresariales/2016/05/ marketing-servicios-significado-caracteristicas/

[4] Cubillos, M. \& Rozo, D. (2009). El concepto de calidad: Historia, evolución e importancia para la competitividad. Revista de la Universidad de La Salle, (48), 80-99.

[5] Decreto Supremo N. 001 (2015, June 9). Reglamento de establecimientos de hospedaje. El Peruano, Normas Legales: 554648.

[6] Francisco, C. de (2014). Tendencias turísticas en el mercado español debidas a segmentos emergentes y potenciales (Undergraduate Thesis). Universidad de Valladolid, Segovia.

[7] Gestión (2017, August 1). Ingresos por canon minero en los gobiernos regionales y locales aumentaron $24.4 \%$ a julio. Gestión. Retrieved from https://gestion.pe/economia/ingresoscanon-minero-gobiernos-regionales-localesaumentaron-24-4-julio-140691

[8] Gestión (2018, July 13). MEM: Canon minero de 2018 fue $70 \%$ superior al del año pasado. Gestión. Retrieved from https://gestion.pe/ economia/mem-canon-minero-2018-70superior-ano-pasado-238429
[9] López, E., Cabrera, Y., López, E. \& Puerto, A. (2018). Calidad percibida en servicios de asistencia al adulto mayor. Medisur, 16(3), 437-463.

[10] Ministerio de Comercio Exterior y Turismo (MINCETUR) (2016). Plan Estratégico Nacional de Turismo 2025. Retrieved from https:// www.mincetur.gob.pe/wp-content/uploads/ documentos/turismo/documentos/PENTUR/ PENTUR_Final_JULIO2016.pdf

[11] Ministerio de Comercio Exterior y Turismo (MINCETUR) (2017). Plan Nacional de Calidad Turística. Retrieved from https://www.mincetur. gob.pe/wp-content/uploads/documentos/ turismo/CALTUR/pdfs_documentos_Caltur/ CALTUR_2017_2025.pdf

[12] Organización de las Naciones Unidas para la Alimentación y la Agricultura (FAO) (2015, July). Plantilla de buenas prácticas. Retrieved from http://www.fao.org/3/a-as547s.pdf

[13] Organización de las Naciones Unidas para la Educación, la Ciencia y la Cultura (UNESCO) (2017). About the MOST Programme. Retrieved from http://www.unesco.org/new/ en/social-and-human-sciences/themes/mostprogramme/about-most/

[14] Organización Mundial del Turismo (OMT) (2002). Turismo: Panorama 2020. Previsiones mundiales y perfiles de los segmentos de mercado. Madrid, Spain: Organización Mundial del Turismo.

[15] Sanabria, P., Romero, V. \& Flórez C. (2014). El concepto de calidad en las organizaciones: una aproximación desde la complejidad. Universidad \& Empresa, 16(27), 165-213. Retrieved from http://www.redalyc.org/ pdf/1872/187241606007.pdf 